... François Mottu, Allgemeinmediziner, Familienarzt in Versoix, Reisender, Tier- und Landschaftsfotograf

\title{
«Augen und Geist fürs Ganze öffnen»
}

\section{Daniel Lüthi}

Text und Bilder

danielluethi@gmx.ch
Der Bildband, der in der Praxis auf dem Pult liegt, zeigt Bären, Wale, Affen und einen Leoparden - und viele andere exotische Tiere. Dazu eindrückliche Landschaftsfotografien aus Kanada, Tansania, Finnland, Russland - und zahlreichen anderen Ländern dieser Welt. «Natur» ist das Thema des Buches, Autor und Fotograf ist François Mottu, «reisender Arzt», wie er sich nennt.

\section{Menschen und ihre Geschichten}

Eigentlich ist Mottu ein sesshafter Mensch. Seit bald dreissig Jahren führt er in Versoix am Genfersee seine Familienpraxis. Das tönt einiges liebevoller und authentischer als der deutsche Begriff «Hausarztpraxis». «Mich interessieren Beziehungen», sagt Mottu, «ich be- treue und begleite während langer Zeit ganze Familien, kenne Grosseltern, Eltern und Kinder, kenne ihre Geschichten.» Die Gesamtschau sei wichtig, die globale Sichtweise, wie er es immer wieder nennt. Das ist eine Erfahrung - das ist aber auch ein medizinisches Credo. Ein Beispiel: Gestern sei eine Patientin mit Rückenschmerzen zu ihm gekommen. Natürlich habe er sich auch direkt darum gekümmert. Entscheidend jedoch sei wahrscheinlich die Diskussion gewesen, die eine starke Arbeitsbelastung der Patientin zutage gefördert habe, eine zu schwere Last eben.

Wenn François Mottu jetzt, rund ein Jahr vor seiner Pensionierung, auf seine vielen Jahre als Hausarzt zurückblickt, ist dies eine seiner hauptsächlichen Beobachtungen: «Alles ist instabiler geworden, und die

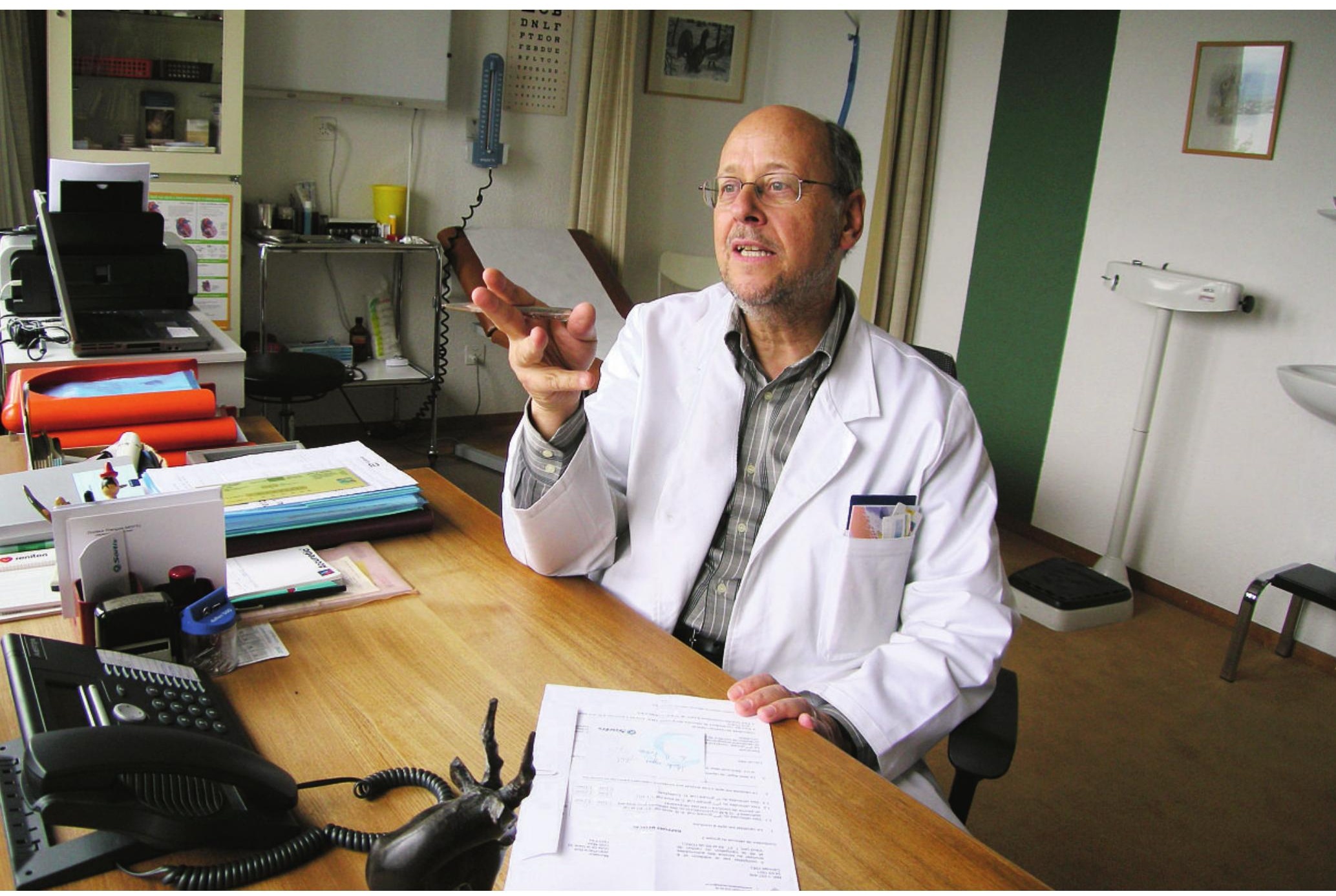


Leute sind klar gestresster als früher. Sie müssen in immer kürzerer Zeit immer mehr produzieren, sie kommen kaum mehr zur Ruhe. Auch dann nicht, wenn sie plötzlich arbeitslos werden.» Rücken- und Verdauungsprobleme sind verbreitete Folgen, auch psychische Krankheiten.

Und sonst, was hat für Veränderungen gesorgt, in all den Jahren? «Das Auftauchen von Google.» Im Internet informieren sich heute viele Patienten, oft wiegen sie sich dann in einer falschen Sicherheit und kritisieren als Folge davon den Arzt. «Es ist ähnlich wie bei einem Horoskop», kommentiert Mottu, «man sieht und glaubt, was man sehen und glauben will.»

\section{Lektionen der Natur}

Der Block, in dem sich die Praxis des Ehepaars Mottu befindet, steht in der Anflugschneise des Flughafens Genf-Cointrin. Direkt vor der Praxis führt die stark befahrene Hauptstrasse vorbei. Viel Lärm also, rundherum. Gleich hinter der Strasse aber, welcher Kontrast, liegt der See. «Er beruhigt mich» sinniert Mottu, «und er verändert sich dauernd - unabhängig, ohne mein Zutun übrigens», wie er schalkhaft beifügt. Doch Schalk ist bloss das eine. Das andere ist die Erkenntnis, die ihm die Natur immer wieder schenkt: Die Macht der Menschen, auch die Handlungsmacht eines Arztes ist beschränkt und steht in einer Relation zu grösseren Dimensionen. «Angesichts der Ewigkeit bin ich bloss ein winziger Punkt.» Oft fahre er mit einem Berufsfischer hinaus, geniesse das Farbenspiel, den kleinen Nebel, die grosse Ruhe. Der Zauber des Augenblicks: Das ist es, was den Arzt auch zum Naturfotografen hat werden lassen.

\section{Die Handlungsmacht eines Arztes} ist beschränkt

Ein Schlüsselerlebnis war die Begegnung mit einem Wolf im Norden Kanadas, nur etwa 800 Kilometer vom Nordpol entfernt. «Er näherte sich unserer Gruppe und schien mit uns zu sprechen», erinnert sich François Mottu. «Ich sehe euch, ich akzeptiere euch», habe er den Eindringlingen mit seinem Verhalten und seinen Blicken mitgeteilt. Unmissverständlich aber auch, dass dies sein Revier sei, und dass der andere, der Betrachter, in dieser Welt bloss vorübergehend geduldet sei. Zur Faszination, die insbesondere Säugetiere auf den Humanmediziner aus Versoix ausüben, gehört, dass auch sie in Familien leben, dass auch sie neugierig sind und dass auch sie kommunizieren können. Überraschende Parallelen zwischen Tier- und Menschenwelt. Dazu gehört laut Mottu auch, dass der Schein oft trügt. So führe die rasche Interpretation einer Körperhaltung oft zu einem voreiligen und falschen Urteil. Ein Bär beispielsweise, der gemütlich und

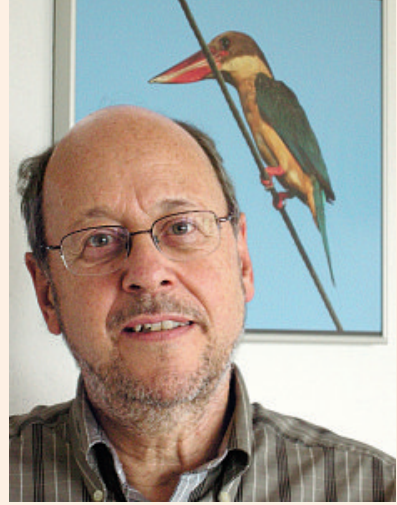

\section{François Mottu}

Dr. med. François Mottu wurde 1947 in Genf geboren, wo er auch die Schulen besuchte und Medizin studierte. Seit 1981 führt er in Versoix bei Genf seine Hausarztpraxis - in einer Praxisgemeinschaft mit seiner Frau Delphine, die Kinderärztin ist. Zusammen mit ihr unternimmt er seit vielen Jahren auch regelmässig Reisen, bei denen ihn vor allem die Beobachtung und das Fotografieren von Tieren interessiert. 2008 hat er den Bildband «Nature sur ordonnance regards d'un médecin voyageur» publiziert. *

Seine medizinische Laufbahn führte François Mottu 1970 nach Leeds in England, wo er sich in Viszeralchirurgie weiterbildete. 1976/77 beschäftigte er sich in Oran (Algerien) mit Pädiatrie. Zweimal war er für das Internationale Komitee des Roten Kreuzes IKRK unterwegs: 1972 besuchte er pakistanische Kriegsgefangene in Indien, 1981 iranische Gefangene im Irak.

1993 bis 2004 wirkte er als Lehrbeauftragter für Hausarztmedizin an der Universität Genf, 1998 bis $\mathbf{2 0 0 2}$ weilte er zweimal pro Jahr in Sarajevo, wo er bosnische Kolleginnen und Kollegen weiterbildete.

François Mottu lebt mit seiner Frau in Versoix. Er ist Vater eines erwachsenen Sohnes und zweifacher Grossvater. Nächstes Jahr wird er pensioniert. Sein Sohn führt die Praxis zusammen mit seiner Frau - beide sind Internisten - als Gruppenpraxis weiter.

friedlich wirke, könne leicht viel gewalttätiger sein, als angenommen.

Für ihn als Arzt sei die Natur Energiequelle und Lehrmeisterin zugleich, sagt Mottu. Der Anblick eines Tieres in der Wildnis lasse ihn die Widerwärtigkeiten des Alltags vergessen und helfe beispielsweise auch, Unzulänglichkeiten und Ungerechtigkeiten unseres 
Gesundheitssystems zu relativieren. Draussen in der Natur könne ein Arzt wieder lernen, vorerst einmal hinzuschauen und zuzuhören und erst dann zu folgern und zu sprechen. In der Natur und zu Hause in der Praxis gehe es darum, nichts zu überstürzen und nicht bloss Einzelheiten, Details wahrzunehmen. Draussen und drinnen, in der Freizeit und während der Arbeit müsse und wolle er «Augen und Geist fürs Ganze öffnen».

In einer Disziplin scheint dieser Allgemeinmediziner besonders spezialisiert zu sein: in der medizinischen Philosophie ...

\section{Realitäten des Alltags}

Bloss eben: Die Wirklichkeit ist oft grobschlächtiger, und auch François Mottu kommt nicht daran vorbei. So regt auch er sich bisweilen über die massiven Einkommensunterschiede bei den Ärztinnen und Ärzten auf, über die schlechtere Stellung der Hausärzte. Mottu

\section{In der Natur und zu Hause in der Praxis geht es darum, nichts zu überstürzen und nicht bloss Einzelheiten, Details wahrzunehmen}

zieht eine handgeschriebene Tabelle aus der Schublade, die gemäss seinen Einträgen nachweist, dass sein Einkommen in den vergangenen bald 30 Jahren absolut gesehen praktisch gleichgeblieben ist, in Relation zu den Ausgaben also klar abgenommen hat. Dabei sei es doch so, dass Hausärzte einiges dazu beitragen würden, im Gesundheitssystem Kosten zu sparen. Über 90 Prozent der Fälle könne er in seiner Hausarztpraxis lösen, sagt Mottu. Dies jedoch werde zu wenig honoriert.
Mit der Selbstdispensation von Medikamenten einen Zusatzverdienst zu erzielen, wie dies in der Deutschschweiz gemacht werde, lehnt der Arzt aus Versoix klar ab. Er sieht nur einen Ansatz: «Der TARMED muss für uns besser werden.»

Deshalb war auch er an jenem denkwürdigen 1. April 2006 auf dem Berner Bundesplatz an der grossen Demonstration. Und deshalb gehörte er pionierhaft zu jenen, die die Hausarztmedizin bereits in der akademischen Ausbildung zu fördern versuchen. «Als Lehrbeauftragter an der Universität Genf konnte ich nicht nur den Studenten etwas weitergeben -, sondern auch den anderen Dozentinnen und Dozenten.» Gemeint ist einmal mehr vor allem die Art, auf Patientinnen und Patienten zuzugehen. Eben: Augen und Geist fürs Ganze zu öffnen ...

\section{Das Gute in der Nähe}

Jetzt also steht François Mottu der sogenannte «Ruhestand» bevor. Er freue sich auf den anderen Rhythmus, sagt er, «alle zwanzig Minuten ein anderer Patient, eine andere Patientin, das ist schon anstrengend». Wohin zieht's einen, der schon so viel gesehen hat? $\mathrm{Zu}$ den Vögeln in Falkland, den Tigern in Tansania oder den Bären in British Columbia? François Mottu schweigt eine Weile. Er betrachtet den aus Holz geschnitzten Kudu auf seinem Pult, der ihn an eine Reise nach Afrika erinnert. Und sagt dann, sein nächstes Projekt liege ganz in der Nähe. Er wolle die Versoix, den kleinen Fluss, porträtieren, der durch das Dorf fliesst und ihm den Namen gegeben hat. Viel gebe es da zu sehen, zwischen Jura und Genfersee, die Farben der vier Jahreszeiten zum Beispiel, verschiedene interessante Tiere auch. Mottu wird wandern und warten. Draussen im Schlafsack übernachten. Er wird hören und schauen und $\mathrm{ab}$ und $\mathrm{zu}$ auch fotografieren.

Und damit einmal mehr den Lauf der Zeit einen kurzen Augenblick lang anhalten.

\section{Die nächste «Begegnung mit ...»}

Am Ende jeden Monats stellt die Schweizerische Ärztezeitung eine Persönlichkeit vor, die sich im Gesundheitswesen engagiert. Im September schildert Daniel Lüthi seine Begegnung mit Heike Gudat, leitende Ärztin des HOSPIZ IM PARK in Arlesheim und Dozentin für Palliative Care an der Universität Basel. 